the TB treatment centre was significant for young and older individuals (OR $0.81 \quad(\mathrm{p}<0.01)$ and OR $0.71 \quad(\mathrm{p}<0.001)$, respectively), indicating lower completion of treatment if the patient lived $>7.3 \mathrm{~km}$ from the treatment centre.

$\mathrm{TB}$ is a rare disease in many high-income countries, which leads to pressures for the pooling of expertise to larger centres [2]. This results in patients being treated in TB centres with higher case loads but greater distances for patients to travel. This study demonstrates that such merging has positive health benefits, as larger centres (load $>26$ cases $\cdot \mathrm{yr}^{-1}$ ) had significantly higher completion rates for $\mathrm{TB}$ treatment for young individuals born outside the UK ( $\sim 50 \%$ of TB patients). However, if centralisation leads to patients having to travel greater distances to receive treatment, then this has negative consequences, as this study shows that all patients born outside the UK $(\sim 65 \%$ of TB patients) were less likely to complete treatment if they had to travel $>7.3 \mathrm{~km}$ to the TB treatment centre. Other studies have indicated the importance of provider experience to treatment outcome [3] but the present study is the first to demonstrate that such experience has a differing impact on subgroups of the population. Previous studies investigating delay in the diagnosis and treatment of $\mathrm{TB}$ have identified the importance of healthcare accessibility [5], but this is the first to be conducted in a high-income country. Additionally, it focuses upon treatment outcome as opposed to diagnosis delay, and examines differential effects amongst population subgroups.

Further research is needed to understand why young individuals born outside the UK are less likely to complete treatment at small TB centres. Migrants may face difficulties in accessing healthcare due to language problems or poor understanding of the health system [3]. They may also require culturally appropriate services [9]. Young migrants may face further difficulties as they require tailored health information. Small TB centres may be unable to address such issues. Many migrants are materially deprived, which, alongside language difficulties [10], may explain why travelling short distances to $\mathrm{TB}$ treatment centres appears difficult. This result was unexpected due to the high-income country setting and the fact that most patients lived close to their TB treatment centre.

This research indicates that moves towards centralisation of TB services, such as implementation of the UK recommendations that clinicians see $\geqslant 10$ TB patients per year [2], should be implemented alongside measures to ensure accessibility of services, targeted to individuals born outside the UK who live far from the treatment centres.

\section{I.R. Lake*, N.R. Jones*, L. Bradshaw" and I. Abubakar",}

*School of Environmental Sciences and "Norwich Medical School, University of East Anglia, Norwich, and "Tuberculosis Section, Centre for Infections, Health Protection Agency, London, UK.

Correspondence: I.R. Lake, School of Environmental Sciences, University of East Anglia, Norwich, UK. E-mail: i.lake@uea. ac.uk

Statement of Interest: None declared.

\section{REFERENCES}

1 WHO. The Stop TB Strategy. Report No. WHO/HTM/TB/ 2006.368. Geneva, World Health Organization, 2006.

2 Dept of Health. Tuberculosis Prevention and Treatment: a Toolkit for Planning, Commissioning and Delivering High-Quality Services in England. London, Dept of Health, 2007.

3 Gardam M, Verma G, Campbell A, et al. Impact of the patientprovider relationship on the survival of foreign born outpatients with tuberculosis. J Immigr Minor Health 2009; 11: 437-445.

4 Wei X, Liang X, Liu F, et al. Decentralising tuberculosis services from county tuberculosis dispensaries to township hospitals in China: an intervention study. Int J Tuberc Lung Dis 2008; 12: 538-547.

5 Storla DG, Yimer S, Bjune GA. A systematic review of delay in the diagnosis and treatment of tuberculosis. BMC Public Health 2008; 8:15.

6 Abubakar I, Crofts JP, Gelb D, et al. Investigating urban-rural disparities in tuberculosis treatment outcome in England and Wales. Epidemiol Infect 2008; 136: 122-127.

7 Office for National Statistics. Indices of Deprivation Across the UK. Newport, ONS, 2009.

8 Bibby P, Shepherd J. Developing a New Classification of Urban and Rural Areas for Policy Purposes. www.statistics.gov.uk/ geography/downloads/Methodology_Report.pdf Date last updated: 2001.

9 Eziefula C, Brown M. The health of recent migrants from resourcepoor countries. Medicine 2010; 38: 60-65.

10 Derose KP, Escarce JJ, Lurie N. Immigrants and health care: sources of vulnerability. Health Aff 2007; 26: 1258-1268.

DOI: 10.1183/09031936.0003621

\title{
Tuberculosis-specific T-cell response after recent treatment and remote cure
}

\section{To the Editors:}

Tuberculosis (TB) remains a major global health problem. A major factor contributing to the current TB epidemic is the resistance of Mycobaterium tuberculosis to first-line anti-TB treatment. Therefore, there is a need for an appropriate and reliable tool to evaluate treatment efficacy.

Several studies using the T-SPOT®.TB assay (Oxford Immunotec Ltd, Oxford, UK) reported a decline in the number of 
spots after successful anti-TB treatment, particularly in the 6kDa early secretory antigenic target (ESAT-6) panel [1-3]; however, to our knowledge, no data have been published on cured TB patients who contracted TB before isoniazid and rifampicin became available. Therefore, the naturally longterm range of ESAT-6- and culture filtrate protein (CFP)-10specific $\mathrm{T}$-cell responses in patients with $\mathrm{TB}$ who did not receive modern anti-TB treatment is not known. Because most patients in prior studies were born in high-burden, TBendemic countries, the T-cell kinetics might be influenced by reinfection. Therefore, the aim of this report is to compare the ESAT-6- and CFP-10-specific T-cell responses in a low-burden country with a low risk of reinfection between patients with a history of active TB before 1955, and patients with recently treated active TB.

Venous blood for T-SPOT ${ }_{\circledast} . T B$ was collected from 24 outpatient subjects with a history of active TB before 1955 (group 1), obtained by clinical history-taking, patients' medical records or documentation of a sanatorium admission. 16 of these patients had been admitted to a sanatorium, two patients underwent surgery and six patients were treated at home with paraaminosalicylic acid therapy or bedrest. Furthermore, 27 patients with recent active TB (group 2) were prospectively enrolled between March and May 2008. Active TB was defined as fulfilling one or more of the following criteria: 1) positive culture for M. tuberculosis $(\mathrm{n}=22) ; 2)$ positive PCR for $M$. tuberculosis $(n=3)$; or 3) a high clinical suspicion of active TB and favourable outcome after receiving anti-TB treatment $(n=2)$. Patients in group 2 had at least two T-SPOT®.TB samples: one before treatment (T0) and one after treatment (T1). The median followup time was 14 months (range 6-44 months).

T-SPOT according to the manufacturer's instructions. The European cut-off value of 6 spot-forming cells (SFCs) was used for the binomial variables for ESAT-6 and CFP-10.

The Mann-Whitney U-test and Chi-squared test were used for between-group comparisons. Wilcoxon's test and McNemar's test were used for within-group analyses. All analyses were performed using SPSS for Windows, version 17.0 (IBM, Amsterdam, the Netherlands).

Patient characteristics for all subjects are shown in table 1. The median time between reported recovery from TB and current $\mathrm{T}$ SРOT®.TB result was 59 yrs. In group 1, 16 (67\%) subjects had positive T-SPOT and one $(4 \%)$ had an indeterminate result. $13(57 \%)$ patients were ESAT-6 positive and $13(57 \%)$ patients were CFP-10 positive. The median (range) number of spots for ESAT-6 and CFP-10 was 7 (0-17) and 18 (0-50) SFCs, respectively (table 2).

In group 2, at T0, 26 (96\%) subjects had positive T-SPOT®.TB results, none of the subjects had a negative result and one (4\%) subject had an indeterminate result. At T1, 23 (85\%) subjects had positive T-SPOT result and none of the subjects had an indeterminate result. The number of subjects with positive T-SPOT®.TB results did not significantly decrease after treatment (nonsignificant).

There was a significant decrease in the number of subjects with positive ESAT-6 results from $25(96 \%)$ at T0 to $17(63 \%)$ at T1

\section{TABLE 1 Baseline characteristics in groups 1 and 2}

\begin{tabular}{lcc} 
Characteristic & Group 1 & Group 2 \\
\hline Subjects & 24 & 27 \\
Age yrs & & \\
$\quad$ At diagnosis & $16(8-22)$ & $43(30-54)$ \\
$\quad$ At first T.SPOT \&. TB & $77(69-85)$ & $43(30-54)$ \\
Males & $17(70.8)$ & $12(44.4)$ \\
Endemic country & $0(0)$ & $13(48.1)$ \\
Smokers & $3(12.5)$ & $2(7.4)$ \\
Immunocompromised & $3(12.5)$ & $0(0)$ \\
Immunotherapy & $6(24.0)$ & $0(0)$
\end{tabular}

Data are presented as $\mathrm{n}$, median (interquartile range) or $\mathrm{n}(\%)$. Group 1: patients with a documented history of tuberculosis before 1955; group 2 : patients with recent active tuberculosis. T.SPOT . TB is manufactured by Oxford Immunotec, Oxford, UK.

$(p=0.008)$. There was no significant difference in the number of subjects with positive CFP-10 results at T0 $(n=25 ; 96 \%)$ and T1 $(\mathrm{n}=22 ; 82 \%)(\mathrm{p}=0.250)$.

There was a significant decline in the median number of spots from 21 (14-42) to 9 (3-32) SFCs for ESAT-6 and from 41 (23-76) to 25 (9-59) SFCs for CFP-10 ( $p=0.003$ and $p=0.020$, respectively).

$\mathrm{T}_{-S P O T} . T B$ results from group 1 were compared with those from group 2 at T0 and T1. There was a significant difference in the number of subjects with positive T-SPOT $₫ . T B(p=0.003)$, ESAT-6 $(\mathrm{p}=0.001)$ or CFP-10 $(\mathrm{p}=0.001)$ results between group 1 and group 2 at T0. There was no significant difference in the number of positive T-SPOT ${ }_{\circledast} . T B(p=0.184)$, ESAT- $6(p=0.643)$ or CFP-10 $(p=0.055)$ results between patients in groups 1 and 2 at T1.

The median number of spots in the ESAT- 6 and CFP-10 panels were significantly different between patients in group 1 and patients in group 2 at $\mathrm{T} 0$ ( $\mathrm{p}=0.001$ and $\mathrm{p}=0.015$, respectively). No differences in median numbers of spots for CFP-10 and ESAT- 6 were observed between groups 1 and 2 at T1.

The correlation between the number of SFCs, and the length of time between treatment completion and blood collection for all patients in groups 1 and 2 was assessed. There was no association between the length of time after treatment completion and the number of spots for ESAT-6 $(\mathrm{p}=0.268)$ or CFP-10 ( $\mathrm{p}=0.637)$.

This study is the first to compare T-cell kinetics in the $\mathrm{T}-S P O T_{\circledR} . \mathrm{TB}$ in $\mathrm{TB}$ patients recently treated with anti-TB treatment, with results in patients with a history of active TB before the introduction of modern anti-TB treatment. Most importantly, patients with a history of "spontaneous" cure (group 1) had comparable T-SPOT®.TB results with patients with recent anti-TB treatment for active TB (group 2 at T1). In contrast, two prior studies showed that therapy resulted in a significant decline in the number of spots, whereas after treatment completion the number of spots did not decline further $[4,5]$.

In patients with cured TB before 1955, T-SPOT®.TB results were positive in $66.7 \%$ after a median survival of 59 yrs. These 
TABLE 2 T-SPOT (TB (Oxford Immunotec, Oxford, UK) results in groups 1 and 2

\begin{tabular}{|c|c|c|c|c|c|c|}
\hline & Group 1 & Group 2 at T0 & p-value ${ }^{\#}$ & Group 2 at T1 & p-value & $\mathrm{p}$-value ${ }^{+}$ \\
\hline Subjects & 24 & 27 & & 27 & & \\
\hline Positive & $16(66.7)$ & 26 (96.3) & 0.003 & $23(85.2)$ & 0.184 & 0.250 \\
\hline Indeterminate & $1(4.2)$ & $1(3.7)$ & & $0(0)$ & & \\
\hline \multicolumn{7}{|l|}{ ESAT-6 } \\
\hline \multicolumn{7}{|l|}{ CFP-10 } \\
\hline Positive & $13(56.5)$ & 25 (96.2) & 0.001 & $22(81.5)$ & 0.055 & 0.250 \\
\hline Median (IQR) & $18(0-50)$ & $41.0(23.2-76.0)$ & 0.015 & $25(9-59)$ & 0.364 & 0.020 \\
\hline
\end{tabular}

Data are presented as $\mathrm{n}$ or $\mathrm{n}(\%)$, unless otherwise stated. ESAT-6: 6-kDa early secretory antigenic target; IQR: interquartile range; CFP: culture filtrate protein. ${ }^{\#}$ : group 1 versus group 2 at T0; ": group 1 versus group 2 at T1; ${ }^{+}$: group 2 at T0 versus group 2 at T1.

results demonstrate that positive T-SPOT®.TB results can persist over time in patients with a history of active TB who did not receive modern anti-TB treatment. This might be the result of a persistent in vivo antigen load in recovered TB patients [6].

Our data show that T-SPOT ${ }_{\circledast} . T B$ results in patients with TB before 1955 were significantly lower than the results in patients with recent TB before treatment (T0). One could hypothesise that after successful treatment, the number of SFCs will decline with time. However, our study and the study of WU-HsiEH et al. [7] did not report an association between the time after recovery and the number of spots.

Moreover, we found that the number of positive T-SPOT®.TB results before (T0) and after (T1) treatment in treated and cured patients with recent active TB were not significantly different. Only $12 \%$ of the individuals with a positive T-SPOT ${ }_{\circledR} . T B$ before treatment converted to negative after treatment. However, we found a significant decline in the number of ESAT-6-positive patients after treatment. Consistent with our results, several studies reported a significant decline in the number of spots in the ESAT-6 panel during or after anti-TB treatment $[2,8]$. The studies by AIKEN et al. [1] in Gambia and DHEDA et al. [9] in South Africa reported not only a significant decline in the number of spots after treatment, but also a significant decline in the amount of positive results for both ESAT-6 and CFP-10, despite high re-infection rates in Gambia and South Africa. One can only hypothesise that these conflicting observations between these studies in high- and low-burden endemic countries might be due to differences in exposure, $M$. tuberculosis strain, genetics, infectious burden, treatment regime and the impact of comorbidity on the immune response to M. tuberculosis [10].

ELISPOT results from patients with active disease were significant higher than those of patients with TB before 1955. This might be due to an acquired state of latent infection with naturally declining kinetics of the T-SPOT ${ }_{\circledast} . T B$ in the last group. After effective anti-TB treatment, the number of spots declined, which might be due to the eradication of $M$. tuberculosis because of effective therapy. Combining these results, it might be suggested that anti-TB treatment accelerates the naturally declining kinetics of the T-SPOT®.TB [6].

This study has some limitations: 1) the limited number of patients; 2) because the T-SРОТв.TB assay was not available in 1955, we could not compare pre- and post-treatment results of these patients; and 3) selection bias could have been introduced, as patients with TB before 1955 who did not recover or even died were not included.

In conclusion, our report is the first to describe the naturally long-term kinetics of the ESAT- 6 and CFP-10 panels of the TSPOT ${ }^{-T B}$ in patients with a history of $\mathrm{TB}$ before the introduction of modern anti-TB treatment. T-SPOT $® . T B$ results do not differ between recently treated TB patients and patient with TB before 1955 in a low-burden, endemic setting. Although the number of spots decline after treatment, the number of patients with T-SPOT ${ }_{\mathbb{R}}$.TB conversion is disappointing, indicating that the T-SPOT priate tool for evaluating treatment outcome and efficacy.

\section{J.P. van Gemert*, S.F.T. Thijsen" and A.W.J. Bossink}

*Emergency Dept, University Medical Centre, Utrecht, and Depts of "Microbiology, and "Pulmonology, Diakonessenhuis, Utrecht, The Netherlands.

Correspondence: J.P. van Gemert, Emergency Dept, UMC Utrecht, Heidelberglaan 100, 3584 CX, Utrecht, The Netherlands. E-mail: anna.vangemert@yahoo.com

Statement of Interest: A statement of interest for A.W.J. Bossink can be found at www.erj.ersjournals.com/site/misc/statement. xhtml

\section{REFERENCES}

1 Aiken AM, Hill PC, Fox A, et al. Reversion of the ELISPOT test after treatment in Gambian tuberculosis cases. BMC Infect Dis 2006; 6: 66.

2 Pathan AA, Wilkinson KA, Klenerman P, et al. Direct ex vivo analysis of antigen-specific IFN- $\gamma$-secreting CD4 $\mathrm{T}$ cells in 
Mycobacterium tuberculosis-infected individuals: associations with clinical disease state and effect of treatment. J Immunol 2001; 167: 5217-5225.

3 Carrara S, Vincenti D, Petrosillo N, et al. Use of a T cell-based assay for monitoring efficacy of antituberculosis therapy. Clin Infect Dis 2004; 38: 754-756.

4 Kobashi Y, Mouri K, Fukuda M, et al. Transitional change in the clinical features of pulmonary tuberculosis. Respiration 2008; 75: 304-309.

5 Bosshard V, Roux-Lombard P, Perneger T, et al. Do results of the T-SPOT.TB interferon- $\gamma$ release assay change after treatment of tuberculosis? Respir Med 2009; 103: 30-34.

6 Mack U, Migliori GB, Sester M, et al. LTBI: latent tuberculosis infection or lasting immune responses to $M$. tuberculosis? A TBNET consensus statement. Eur Respir J 2009; 33: 956-973.
7 Wu-Hsieh BA, Chen CK, Chang JH, et al. Long-lived immune response to early secretory antigenic target 6 in individuals who had recovered from tuberculosis. Clin Infect Dis 2001; 33: 1336-1340.

8 Ribeiro S, Dooley K, Hackman J, et al. T-SPOT.TB responses during treatment of pulmonary tuberculosis. BMC Infect Dis 2009; 9: 23.

9 Dheda K, Pooran A, Pai M, et al. Interpretation of Mycobacterium tuberculosis antigen-specific IFN- $\gamma$ release assays (T-SPOT.TB) and factors that may modulate test results. J Infect 2007; 55: 169-173.

10 Elias D, Britton S, Kassu A, et al. Chronic helminth infections may negatively influence immunity against tuberculosis and other diseases of public health importance. Expert Rev Anti Infect Ther 2007; 5: 475-484.

\section{Tracheal granuloma pyogenicum with erlotinib treatment for lung cancer}

\section{To the Editors:}

We present the case of a 64 yr-old ex-smoker (20 pack-yrs) with a squamous cell carcinoma of the lung who developed a tracheitis and granuloma pyogenicum during treatment with erlotinib. Among the specific side-effects of epidermal growth factor receptor (EGFR) inhibitors, paronychia with granuloma pyogenicum has been reported frequently; however, granuloma pyogenicum has never been described in the trachea with erlotinib treatment.

Monoclonal antibodies against the EGFR (e.g. cetuximab) and EGFR tyrosine receptor inhibitors (TKIs) (e.g. gefinitib and erlotinib) have been shown to improve treatment outcomes in nonsmall cell lung cancer. Generally, these agents are better tolerated than classical cytotoxic agents showing a different toxicity profile. Specific side-effects of EGFR-targeting agents include diarrhoea and particularly skin toxicity, such as acneiform eruptions, xerosis, eczema, fissures, teleangiectasia, hyperpigmentation, hair changes and paronychia with granuloma pyogenicum [1]. Skin toxicity has been associated with EGFR inhibitor antitumor activity [1].

A 64 yr-old male was diagnosed with squamous cell carcinoma of the right upper lobe and was treated with a right upper lobectomy in December 2002 (pathological (p)T1 pN1 (three positive lymph nodes out of 33 removed)). No adjuvant treatment was given at that time. In 2007, the patient was treated with a right lower lobe resection due to recurrence; the histology confirmed a squamous cell carcinoma (retreatment classification (r)pT1b rpN0 R0). In 2009, he developed a second recurrence in the right mediastinal lymph nodes (stations $4 \mathrm{R}$ and 7) and was treated with a rest-pneumonectomy, showing three out of three positive lymph nodes with angiosis carcinomatosa (rpT1a rpN2 R0). The patient received four cycles of adjuvant cisplatin/vinorelbine, but developed progression in the mediastinal lymph nodes immediately after chemotherapy. Thus, palliative treatment with erlotinib $(150 \mathrm{mg})$ was initiated in
October 2009. He developed acneiform skin lesions of moderate intensity, as typically seen during therapy with a TKI, and achieved disease stabilisation with erlotinib for almost a year. In June 2010, he developed a vigorous dry cough that was refractory to various treatments, including codeine, steroids (systemic and inhaled) and $\mathrm{H}_{2}$ blockers.

In order to further investigate the cause of the cough, a bronchoscopy was performed, which showed a severe acute and chronic tracheobronchitis with significant breath-dependent tracheobronchial collapse and multiple white granular mucosal lesions of the distal trachea, which were biopsied (fig. 1).

To our surprise, the histopathological work-up showed no evidence of tumour, which was initially suspected, but small nodular lesions with granulation tissue and fibrinous material at the surface (which are white in colour when viewed on endoscopy), consistent with granuloma pyogenicum (fig. 2).

As no other explanation for the tracheal granuloma pyogenicum and the agonising cough was identified, treatment with erlotinib was stopped in July 2010, resulting in improvement of the cough within 2 weeks and complete remission of the granulomas in the follow-up bronchoscopy 6 weeks later (fig. 3).

5 weeks later, due to the progression of the mediastinal lymph nodes, a tracheal stent was placed; during the course of the disease, mediastinal radiotherapy with 30 Gy was performed due to further lymph node progression. The patient is currently undergoing further chemotherapy due to systemic progression but has had no recurrence of cough attacks.

Granuloma pyogenicum (also known as eruptive haemangioma, granulation tissue-type haemangioma, granuloma gravidarum and lobular capillary haemangioma [2]) is a polypoid form of capillary haemangioma. It is hypothesised to be a nonspecific tissue reaction seen after various insults but pathogenesis is controversial (neoplasm versus reactive hyperplasia) [3]. It occurs on either the skin or at the mucosal 